\title{
Estimation of Sea Surface Temperature from Space
}

\author{
D. ANDING and R. KAUTH, Institute of Science and Technology. The University of Michigan. Ann Arbor. Michigan
}

\begin{abstract}
A procedure is derived for obtaining improved estimates of water surface temperature by means of spatially scanning space-borne systems which would perform simultaneous radiometric measurements in two wavelength intervals in the thermal infrared atmospheric-window spectral regions. The procedure should reduce errors in the estimate of water surface temperature caused by haze and water vapor effects from approximately $=2.0^{\circ} \mathrm{C}$ to approximately $\pm 0.15^{\circ} \mathrm{C}$.
\end{abstract}

\section{Introduction}

A knowledge of the distribution of water surface temperature over all bodies of water on the earth's surface is of significant importance to a number of scientific communities. Such information would be particularly useful in locating various species of fish, in spatially mapping ocean and lake currents, and in estimating the exchange of thermal energy between the water surface and the atmosphere. In general, the more precise the knowledge of the temperature distribution, the more useful are the data. However, the greater the precision desired, the higher is the cost of achieving that objective. Stevenson (1970), a representative of the scientific community, has stated that estimates of relative temperature within $\pm 1^{\circ} \mathrm{C}$ at a spatial resolution of approximately $1 \mathrm{~km}$ would be satisfactory. Another requirement of the scientific community is that the time period between the collection of the data and its dissemination in a useful form be of the order of days. In essence, an accurate near real-time synoptic view of the earth's water is desired.

Although such an objective could be satisfied in a number of ways, a feasible approach would be to utilize a spatially scanning space-borne system which includes a computerized datareduction technique to facilitate rapid conversion of the measured data into a usable form, such as water surface temperature maps. Fortunately, most of the knowledge required to design such a system is presently available from the results of past analysis and measurement programs. In particular, spatially scanning space-borne systems have been flown, such as the Nimbus III medium-resolution infrared radiometer (MRIR), which had the capability of providing estimates of water surface temperature on a global basis. However, since these systems did not have the capability of compensating for the effects of the atmosphere, temperature estimates with errors of the order of $\pm 2^{\circ} \mathrm{C}$ were the best that could be obtained. Also, these systems had a ground resolution greater than $6 \mathrm{~km}$ and were not designed for rapid, automatic data reduction. The problem is to determine the system modifications required to satisfy the stated objectives. The most important modification is the incorporation of techniques to compensate for atmospheric effects in order to obtain improved estimates of water surface temperature. This paper presents and discusses a technique for achieving this objective.

Theoretical basis of remote Sea Temperature Measurement at Thermal Infrared Wavelengths

The spectral radiance emitted by a greybody at wavelength $\lambda$ is given by

$$
L_{\lambda} \mathbf{E}=\varepsilon(\lambda) L_{\lambda}{ }^{\mathrm{b}}(T)
$$

where $\varepsilon(\lambda)$ is the spectral emissivity of the greybody, and $L_{\lambda} \mathrm{b}(T)$ is the radiance emitted by a blackbody. The latter is represented as

$$
L_{\lambda}{ }^{\prime}(T)=\frac{2 c^{2} h}{\lambda^{5}[(\exp h c / \lambda k T)-1]}
$$

where $T$ is the temperature of the blackbody, $c$ is the velocity of light, $h$ is Planck's constant, $k$ is Boltzmann's constant, and $\lambda$ is the wavelength.

It is clear from these expressions that, if the emissivity is known, the greybody temperature can be determined by measuring the emitted spectral radiance and inverting equation (1). The application of such a measurement procedure in determining the temperature of a water surface exposed to the atmosphere is more complex. The spectral emissivity of a sea surface has a maximum value of approximately 0.98 , which occurs near $11 \mu \mathrm{m}$. Consequently, as one attempts to measure the emitted radiation, some sky radiation is reflected from the water surface and collected by the infrared sensor. Also, since water does not become opaque to infrared radiation at thermal wavelengths until a depth of approximately $0.10 \mathrm{~mm}$, some of the measured radiation emanates from water below the surface, which generally has a slightly different temperature. Therefore the temperature derived from a measurement of the radiance at the surface is the temperature of a greybody (whose emissivity is equal to the emissivity of the water surface) that yields an equivalent value of radiance (i.e., the "equivalent greybody temperature"). It is different from the actual surface temperature, the degree of difference depending on the magnitude of the reflected radiation and the temperature gradient near the surface.

The present analysis is not concerned with the relationship between the equivalent greybody temperature and the actual temperature, however, but only with the effect of the atmosphere on the equivalent greybody temperature derived from a radiometric measurement performed at satellite altitudes. Therefore all future references to water surface temperature denote the equivalent greybody temperature that would be derived from a radiance measurement at the surface.

\section{Effect of the Atmosphere on Measured Radiance}

Before reaching a space-borne sensor, the spectral radiance emanating from the water surface is attenuated by atmospheric constituents, such as clouds, haze, and absorbing gases. These atmospheric constituents also emit and scatter radiant energy which contributes to the total signal received by the sensor. The central problem in measuring accurately the water surface temperature from space lies in determining the extent to which such effects can be observed and compensated.

The atmosphere may be free of clouds or may contain clouds which are either opaque or semitransparent to the surface radiation. If the field of view of a measuring instrument is completely filled with an opaque cloud, no radiation from the water surface reaches the sensor, and a water surface temperature determination is impossible. However, if the surface is only 
partially obscured by a cloud or completely obscured by a semitransparent cloud, a surface temperature estimate can be made. The optimum technique for detecting the presence of clouds and for making estimates of water temperature when the field of view contains clouds will be the subject of a forthcoming paper. This paper considers only the special case of atmospheres containing normal molecular constituents and haze.

In a clear atmosphere the spectral radiance leaving the top of the atmosphere is the sum of two sources of radiation: (i) the radiance from the water surface, as attenuated by the infrared-active molecules $\left(\mathrm{H}_{2} \mathrm{O}, \mathrm{CO}_{2}, \mathrm{O}_{3}, \mathrm{CH}_{4}\right.$, and $\left.\mathrm{N}_{2} \mathrm{O}\right)$ and atmospheric haze, and (ii) the radiance emitted by these atmospheric constituents. Mathematically, this spectral radiance, $L_{\lambda}$, is given by

$$
L_{\lambda}=L_{\lambda}^{\mathrm{g}}(T)-\bar{c}(\lambda)+L_{\lambda}^{\mathrm{a}}
$$

where $L_{\lambda} \mathrm{B}(T)$ is the equivalent greybody radiance at the water surface, $\tau(\lambda)$ the atmospheric spectral transmittance, $T$ the greybody temperature, and $L_{\lambda}{ }^{a}$ is the spectral radiance emitted by the atmosphere.

To demonstrate the magnitude of the effect of atmospheric absorption and emission on the spectral radiance leaving the top of the atmosphere, consider the radiance spectra given in Fig. 1. The solid curve is the spectral radiance from a water surface with a temperature of $290^{\circ} \mathrm{K}$, as viewed from $100 \mathrm{~km}$ through a typical noncloudy atmosphere at an angle of $60^{\circ}$ with the nadir. The dotted curves designate the spectral radiance that would be observed in the absence of an intervening atmosphere for water temperatures of $290^{\circ} \mathrm{K}$ and $285^{\circ} \mathrm{K} .^{\prime}$ The spectral regions in which the effect of the atmosphere is the greatest (i.e., near $6.3 \mu \mathrm{m}, 9.6 \mu \mathrm{m}$, and $15 \mu \mathrm{m}$ ) correspond respectively to the molecular absorption bands of water vapor, ozone, and carbon dioxide. The atmosphere has a lesser effect between these absorption bands, in the so-called atmospheric windows, the minimum effect occurring in the vicinity of $11 \mu \mathrm{m}$. However, even in this clearest atmospheric window, a temperature approximately $2^{\circ} \mathrm{C}$ less than the surface temperature would be inferred from space-borne radiance measurements if the effect of the atmosphere were neglected.

The difference between the temperature derived from spaceborne measurements and the water surface temperature depends both on the distribution of atmospheric water vapor and temperature and on the nadir viewing angle. For nadir angles corresponding to near-horizon viewing and for very moist atmospheres, the spectral radiance at $11 \mu \mathrm{m}$ would correspond to temperatures approximately $4^{\circ} \mathrm{C}$ cooler than the surface temperature. Even at near-nadir viewing through relatively dry, clear atmospheres, the temperature at $11 \mu \mathrm{m}$ would be approxi-

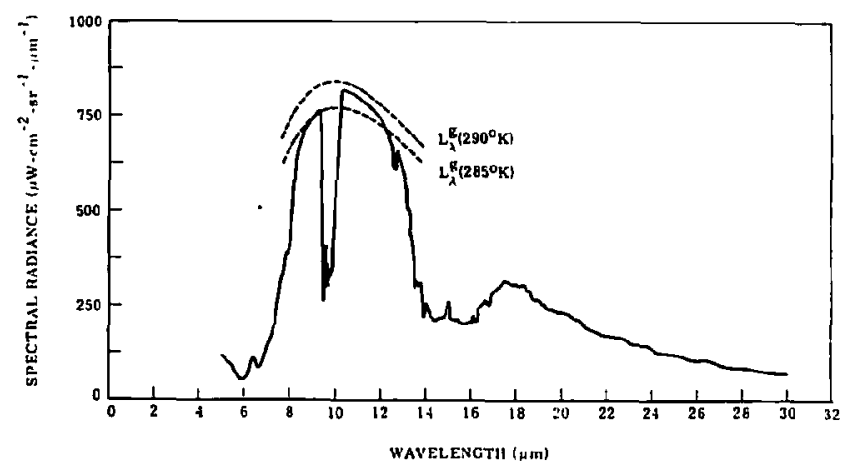

Fig. 1. Spectral radiance at $100-\mathrm{km}$ altitude for a water surface temperature of $290^{\circ} \mathrm{K}$. Nadir viewing angle $=60^{\circ}$.

Remote Sensing of Environment 1 (1970), 217-220 mately $1^{\circ} \mathrm{C}$ cooler than the surface temperature. Therefore estimates of the water surface temperature within $\pm 1{ }^{\circ} \mathrm{C}$ can be achieved only by correcting for the effects of atmospheric absorption and emission. A procedure devised to compensate for these effects is a simple application of multiband remote sensing techniques requiring simultaneous measurement of the radiance in two specially chosen wavelength regions, one in each of the two atmospheric windows (i.e., near $9.0 \mu \mathrm{m}$ and (l $\mu \mathrm{m})$.

\section{Compensation of Atmospheric Effects with a System Utilizing} Two Spectral Bands

In the two window regions, 7-9.5 $\mu \mathrm{m}$ and $10-12 \mu \mathrm{m}$, the only atmospheric constituent which significantly absorbs and emits radiation is water vapor, the absorption primarily resulting from the far wings of the intense spectral absorption lines in the $6.3-\mu \mathrm{m}$ absorption band and those in the rotational water band (which begins to absorb strongly beyond $15 \mu \mathrm{m}$ ). The absorption near $9 \mu \mathrm{m}$ is greater than that near $11 \mu \mathrm{m}$; therefore the temperature inferred from radiance measurements near $9 \mu \mathrm{m}$ is less than that inferred from measurements near $11 \mu \mathrm{m}$ (Fig. 1). Since the physical process which causes the absorption and the atmospheric emission in both window regions is the same, with the effect near $9 \mu \mathrm{m}$ simply a slight magnification of the effect near II $\mu \mathrm{m}$, a simultaneous measurement of the radiance in each window region should provide the necessary data to estimate the magnitude of water vapor absorption and emission. The problem involved (i) establishing the existence of two or more such spectral bands, and (ii) specifying the bands which would yield the optimum results.

The first step in the analysis was to obtain the relationship between the spectral radiance leaving the top of the atmosphere and the altitude distribution of water vapor and atmospheric temperature. Second, by use of this relationship, the existence of two spectral bands which would provide the necessary data to estimate the magnitude of water vapor absorption and emission was established. Finally, the optimum spectral bands for satisfying the stated objectives were selected.

Because of the complex relationship between the spectral radiance leaving the top of the atmosphere and the distribution of atmospheric water vapor and temperature, the relationship was empirically established by calculating the spectral radiance at $100-\mathrm{km}$ altitude as a function of nadir viewing angle and water surface temperature for a number of different atmospheric water vapor and temperature distributions. An existing spectral radiance computer program, described by Anding and Kauth (1969), was used in these calculations. This computer program has the capability of calculating the spectral radiance from 5 to $30 \mu \mathrm{m}$, including the effects of water vapor, ozone, carbon dioxide, methane, nitrous oxide, and haze. Calculations can be performed for any nadir viewing angle and any distribution of atmospheric parameters. Since this analysis was concerned only with the effect of the atmosphere on the spectral radiance in the atmospheric window spectral regions, all atmospheric gas concentrations were held constant at representative average values, and water vapor and temperature were varied over a range of values. The actual temperature and water vapor altitude profiles used in these calculations are presented, respectively, in Figs. 2 and 3. They are representative of extreme conditions for summer and winter seasons for an at-

${ }^{1}$ For this case, $\tau(\lambda)=1.0$ and $L_{\lambda}{ }^{a}=0$. Therefore the observed radiances are respectively given by $L_{\lambda}=L_{\lambda}^{g}\left(290^{\circ} \mathrm{K}\right)$ and $L_{\lambda}=L_{\lambda}^{g},\left(285^{\circ} \mathrm{K}\right)$.

D. Anding and R. Kauth 


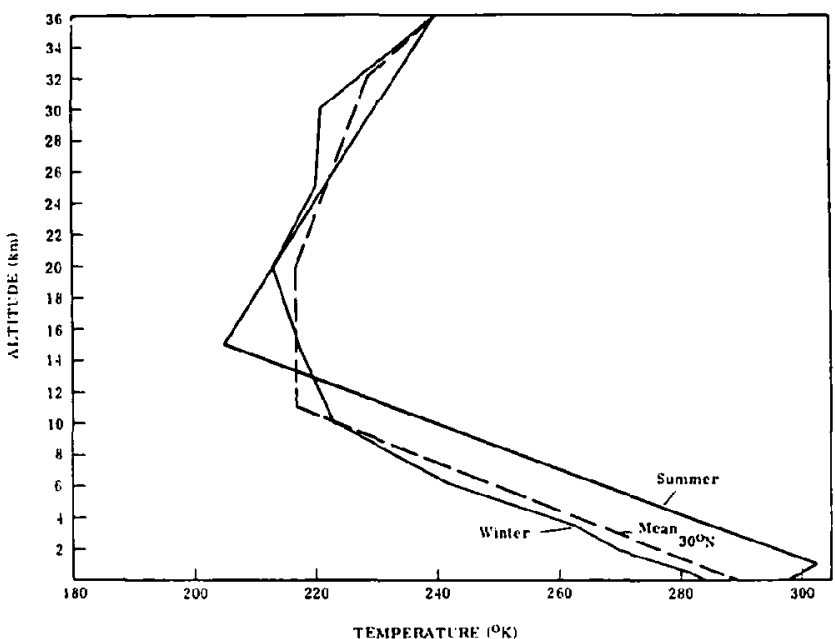

Fig. 2. Temperature profiles.

mosphere at latitude $30^{\circ} \mathrm{N}$. Although these profiles do not bound all atmospheric conditions which could exist over the entire globe, they do encompass a sufficient range of values to determine the desired relationship between spectral radiance and atmospheric state. From these water vapor and temperature data, five model atmospheres were obtained by selecting five pairs of temperature and water profiles as shown in Table 1.

Table I

\begin{tabular}{lll}
\hline $\begin{array}{l}\text { Model atmospheric } \\
\text { name }\end{array}$ & $\begin{array}{l}\text { Temperature } \\
\text { profile }\end{array}$ & Water profile \\
\hline Summer wet & Summer & Summer wet \\
Summer dry & Summer & Summer dry \\
Winter wet & Winter & Winter wet \\
Winter dry & Winter & Winter dry \\
Mean, 30'N & Mean & Mean \\
\hline
\end{tabular}

For each model atmosphere, spectral radiance calculations were performed for three nadir angles $\left(0^{\circ}, 60^{\circ}, 75^{\circ}\right)$ and five water surface temperatures $\left(280^{\circ}, 285^{\circ}, 290^{\circ}, 295^{\circ}\right.$, and $\left.300^{\circ} \mathrm{K}\right)$. Thus a total of 75 spectra were calculated. The values of spectral emissivity of the water surface and the degree of dependence of emissivity on nadir viewing angle were taken from the work of Buettner and Kern (1965).

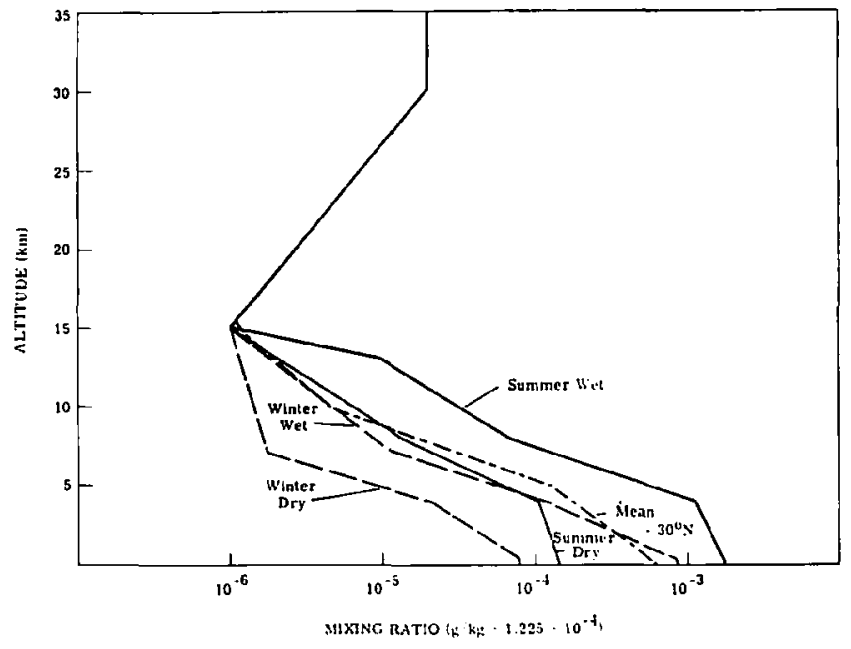

Fig. 3. Water vapour profiles.

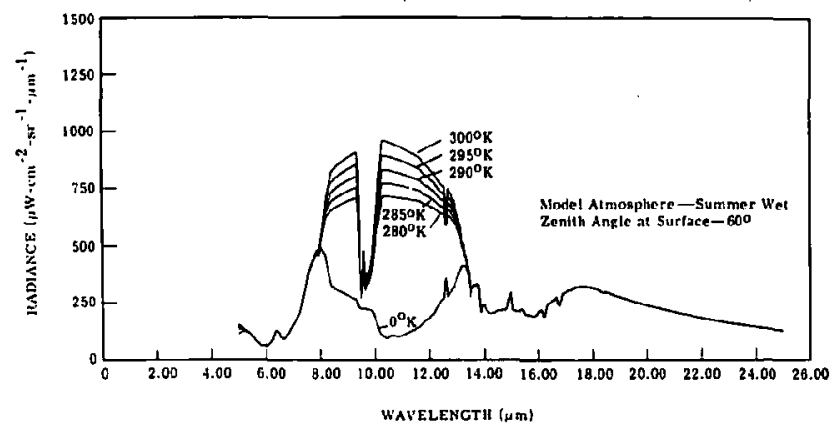

Fig. 4. Upwelling spectral radiance at $100 \mathrm{~km}$ with sea surface temperature as a parameter.

An example of the spectral radiance data calculated is given in Fig. 4. The data represent the upwelling spectral radiance at $100 \mathrm{~km}$ at a nadir angle of $60^{\circ}$ for a summer-wet atmosphere and six water surface temperatures. Each of the radiance spectra is the radiance emitted by a greybody at a temperature equal to the water-surface temperature, modified by the molecular absorption and emission bands of the atmosphere. The $0^{\circ} \mathrm{K}$ surface temperature was included to reveal the magnitude of the spectral radiance emanating only from the atmosphere. Near the center of the intense absorbing regions (i.e., the $6.3-\mu \mathrm{m}$ $\mathrm{H}_{2} \mathrm{O}$ band and the $15-\mu \mathrm{m} \mathrm{CO} \mathrm{CO}_{2}$ band), the spectra are the same for all water surface temperatures because the atmosphere is opaque in these spectral regions.

The validity of the use of the calculated spectra for the present analysis was established by comparing calculations of spectral radiance with data obtained with the Nimbus III Infrared Interferometer Spectrometer (IRIS) for known atmospheric conditions. A representative result of such comparisons is shown in Fig. 5. Although in some spectral regions minor differences are observable, they are negligible in the atmospheric-window regions.

The relationship between the spectral radiance near $9 \mu \mathrm{m}$ and that near $11 \mu \mathrm{m}$ was empirically derived from the calculated spectra. Each of the calculated radiance spectra was spectrally smoothed to various resolutions, each resolution element being narrow enough to remain within an atmospheric window but broad enough to accept sufficient radiant power when implementing a satellite-borne scanning radiometer. Specifically, the spectra were smoothed to three different resolutions, $0.5,1.0$, and $1.5 \mu \mathrm{m}$, and the radiance values were obtained every $0.1 \mu \mathrm{m}$ throughout each window region. From the smoothed data, all possible pairs of radiance values were selected, one value from the $9-\mu \mathrm{m}$ window and one from the $11-\mu \mathrm{m}$ window. For each pair of values, one value of radiance was

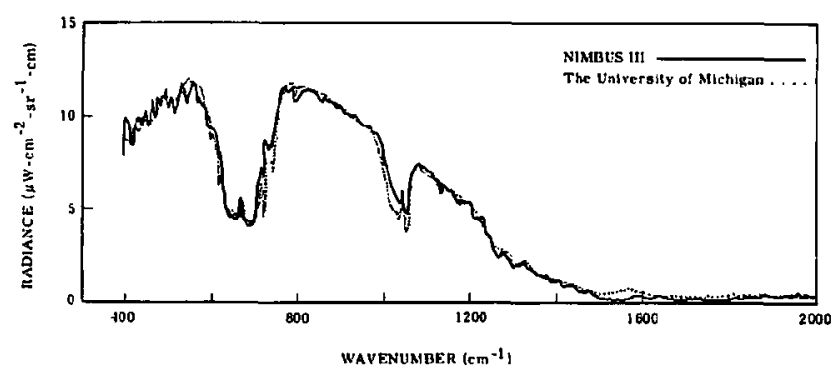

Fig. 5. Comparison of the University of Michigan calculations and the satellite-borne measurements.

Remote Sensing of Environment 1 (1970), 217-220 


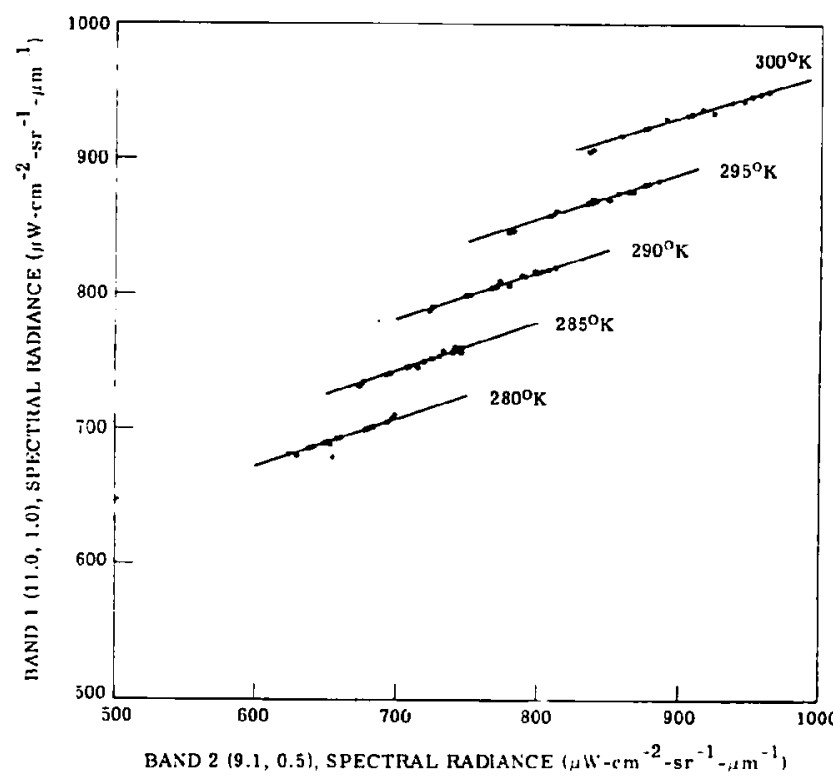

Fig. 6. Spectral radiance in band 1 vs that in band 2 as a function of at mospheric state. Parameters = zenith angle at surface and sea surface temperature.

plotted versus the other, each data point corresponding to a single atmospheric state, a particular nadir viewing angle, and a given water surface temperature. An example of one such plot is shown in Fig. 6. Each spectral band is denoted by its center wavelength and spectral bandpass $\left(\lambda_{c}, \Delta \lambda\right)$. The values for the band pair represented are, respectively, $(9.1,0.5)$ and $(11.0,1.0)$. The radiance values for each temperature are well represented by straight lines, which were chosen to minimize the rms orthogonal error. For many of the band pairs, nonlinearities were observed and, in most cases, the scatter in the data points was extremely large. The result shown is for the band pair which gave the best results in regard to linearity and minimum scatter.

The interpretation of the results obtained is straightforward. Given the radiance in one spectral band and the water surface temperature, the spectral radiance in the other band is accurately predictable. In essence, the amount of atmospheric absorption and emission can be predicted. Conversely, given the radiance in each of the two spectral bands for any arbitrary distribution of atmospheric water vapor and temperature, the water surface temperature can be accurately estimated. The existence of a band pair showing linearly related radiance values is not surprising. Since the physical process which causes the absorption and the atmospheric emission in both spectral regions is the same, with the effect in the $9.1-\mu \mathrm{m}$ band simply a slight magnification of that near $11 \mu \mathrm{m}$, a high correlation between the radiance values was expected. The linearity results from the fact that the atmospheric absorption and emission in both spectral regions are small and well represented by linear functions.

The unique feature of the result is that the series of nearly parallel, equally spaced lines provides a basis for a simple datareduction algorithm, which is but a linear interpolation between the equitemperature lines. Had the relationship between the two channel radiance values been defined by a more complicated function, the two channel measurements could still provide accurate estimates of water surface temperature. The data-reduction algorithm would, however, be more complex.

To demonstrate the magnitude of the errors resulting from the use of this algorithm to estimate the water surface temperature, a surface temperature was calculated for each of the 75 radiance pairs plotted in Fig. 6. For each data point the difference between the estimated temperature $T_{i}$ and the water surface temperature $T$, was calculated. The rms error $E$ in the estimated surface temperatures was then calculated by the formula

$$
E=\sqrt{\frac{1}{N} \sum_{i=1}^{N}\left(T_{i}-T_{s}\right)^{2}}
$$

where $N$ is the total number of data points (here $N=75$ ). For the band pair given in Fig. 6, the error was determined to be $\pm 0.15^{\circ} \mathrm{C}$, a significant improvement over an error of $\pm 2^{\circ} \mathrm{C}$, the best obtainable with a single-band system.

\section{Summary and Conclusions}

This analysis has demonstrated that, by performing radiometric measurements simultaneously in two spectral bands located in the thermal infrared atmospheric-window regions, the effects of water vapor absorption and emission can be almost completely compenated without the aid of meteorological support data. The $\pm 0.15^{\circ} \mathrm{C}$ error in the temperature estimate resulting from atmospheric effects, coupled with a noise equivalent temperature of approximately $0.4^{\circ} \mathrm{C}$ (representative of state-ofthe-art systems), indicates that temperature estimates well within the stated objectives of $\pm 1.0^{\circ} \mathrm{C}$ are obtainable for noncloudy atmospheres. By the use of additional spectral bands it is possible to indicate automatically the presence of clouds within the field of view and to estimate the water surface temperature in the presence of partial obscuration. The number of spectral bands, the band centers, and the bandwidths required to achieve this goal will be delineated in a forthcoming paper.

\section{Acknowledgments}

The authors gratefully acknowledge the contribution of Mr. Glen Larson of NASA-ERC in defining the objectives of this effort.

\section{References}

Anding, D., and R. Kauth (1969), Atmospheric modeling in the infrared spectral region: Atmospheric effects on multispectral sensing of sea-surface temperature from space, Report 2676-1-P, Willow Run Laboratories, Institute of Science and Technologly, The University of Michigan, Ann Arbor, Michigan.

Buettner, K. J., and C. D. Kern (1965), The determination of infrared emissivities of terrestrial surfaces, J. Geophys. Res., 70 (6).

Stevenson, R. E. (1970), Oceanographic applications and limitations of satellite remote sensors, Bull. Am. Inst. Aeronaut. Astronaut., 7 (2).

\section{Received July 27, 1970}

The research described in this paper was performed under Contract No. NAS12-2117, sponsored by the Electronics Research Center, National Aeronautics and Space Administration. 